\title{
La formation hospitalo- universitaire spécialisée actuelle en chirurgie orale en France
}

\section{Current specialized hospital-university training in oral surgery in France}

\section{Résumé}

MOTS-CLEFS :

- Diplôme-Chirurgie orale-Formation

\section{KEYWORDS:}

- Academic degree-Oral surgery-Training cursus

$\operatorname{AOS} n^{\circ} 282-2017$
La formation spécialisée en chirurgie orale française a fait un grand bond en avant avec la création du Diplôme d'Études Supérieures en Chirurgie Orale en 2011. Ce diplôme est vraiment original puisqu'il possède une double entrée, médicale par l'intermédiaire de l'examen classant national et dentaire par celui de l'internat en Odontologie. De fait, ces nouveaux praticiens qualifiés en chirurgie orale vont d'une part remplacer les médecins stomatologistes du fait de la fermeture du DES de Stomatologie et d'autre part succéder aux chirurgiens dentistes titulaires du diplôme d'études supérieures en chirurgie buccale. Ce nouveau diplôme va permettre de former des praticiens ayant un champ d'activité élargi de la médecine orale et de la chirurgie orale. Une nouvelle ère s'ouvre donc pour cette spécialité qui va se nourrir de ses deux filières d'origine, qui va murir et qui va proposer une offre de soins de haute qualité dans un secteur très déficitaire.

\author{
Abstract
}

The specialist training in French oral surgery made a big jump(leap) forward with the creation of the Diploma of Higher education in Oral Surgery in 2011. This diploma is really original because it possesses a double entrance(entry), medical through the national and dental classifying examination by that of the boarding school(internship) in Odontology. Actually, these new practitioners qualified in oral surgery are going to replace on one hand the doctors stomatologists because of the closure(lock) of the DES de Stomatologie and on the other hand to succeed the dental surgeons holders of the diploma of higher education in oral surgery. This new diploma is going to allow to train(form) practitioners having a field of activity widened by the oral medicine and by the oral surgery. A new era thus opens for this speciality which is going to feed on its two sectors(networks) of origin, which is going to murir and which is going to propose on offer of care of high quality in a very overdrawn sector.

\footnotetext{
$\begin{aligned} & \text { Philippe LESCLOUS, PU-PH : Faculté de Chirurgie Dentaire de Nantes. } \\ & \text { philippe.lesclous@univ-nantes.fr } \\ & \text { Benoît LEFÈVRE, PU-PH : Faculté de Chirurgie Dentaire de Reims. }\end{aligned}$
Bental
} 


\section{INTRODUCTION}

La formation nationale hospitalo-universitaire accessible aux chirurgiens dentistes labélisée chirurgie buccale puis orale (terme aujourd'hui usité par souci d'harmonisation européenne) est d'apparition assez récente en France. Auparavant, ce type de formation était à l'initiative de quelques facultés de chirurgie dentaire sous la forme de diplômes universitaires. Elle a débuté par la création du Diplôme d'Etudes Supérieures de Chirurgie Buccale (DESCB) le 2 août 1989. Ce diplôme d'une durée de 4 ans, apoptotique depuis la création d'un troisième cycle long spécialisé en chirurgie orale en 2011 en vue de l'obtention du Diplôme d'Etudes Supérieures en Chirurgie Orale (DESCO), n'est plus organisé depuis deux ans. L'objectif de la création successive de ces diplômes est d'offrir une de formation de haute qualité dans le domaine de la médecine orale et de la chirurgie orale visant à combler un besoin de santé publique croissant dans ce domaine.

Cet article a pour buts d'une part d'informer le lecteur $\mathrm{du}$ parcours de formation actuelle en chirurgie orale en France et d'autre part, d'illustrer à l'aide de cas cliniques la diversité de la formation clinique de ces étudiants de spécialité (Figures 1 à 6).
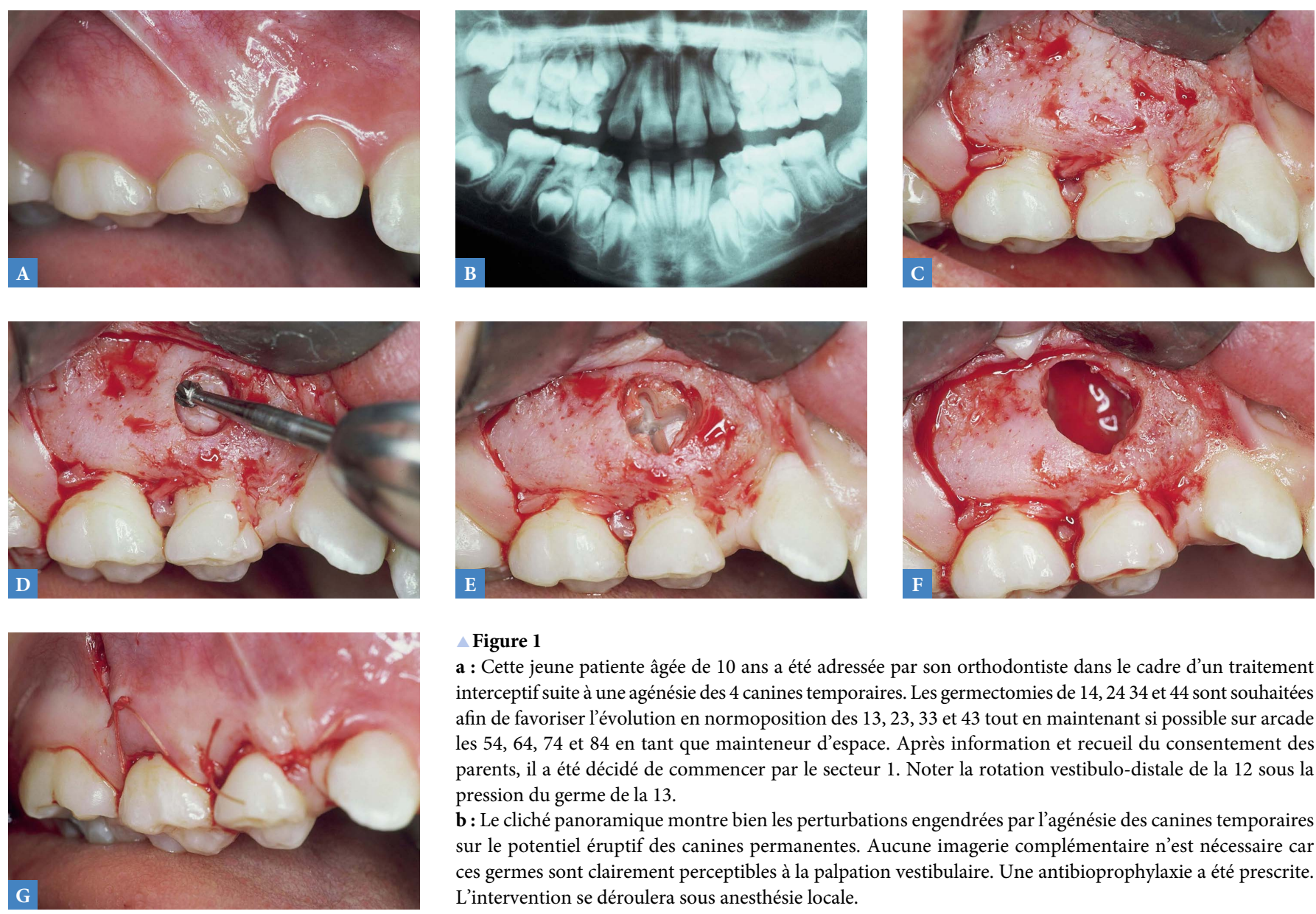

$\triangle$ Figure 1

a : Cette jeune patiente âgée de 10 ans a été adressée par son orthodontiste dans le cadre d'un traitement interceptif suite à une agénésie des 4 canines temporaires. Les germectomies de 14,2434 et 44 sont souhaitées afin de favoriser l'évolution en normoposition des 13,23, 33 et 43 tout en maintenant si possible sur arcade les 54, 64, 74 et 84 en tant que mainteneur d'espace. Après information et recueil du consentement des parents, il a été décidé de commencer par le secteur 1 . Noter la rotation vestibulo-distale de la 12 sous la pression du germe de la 13.

b : Le cliché panoramique montre bien les perturbations engendrées par l'agénésie des canines temporaires sur le potentiel éruptif des canines permanentes. Aucune imagerie complémentaire n’est nécessaire car ces germes sont clairement perceptibles à la palpation vestibulaire. Une antibioprophylaxie a été prescrite. L'intervention se déroulera sous anesthésie locale.

c: Un lambeau vestibulaire trapézoïdal de pleine épaisseur est récliné. La voussure témoignant de la présence du germe de 14 est bien visible. Le plan muqueux palatin reste fixe.

d: Une trépanation osseuse de petit diamètre est pratiquée à la fraise boule acier montée sur pièce à main à l'aplomb de la 54.

e: Une fois repéré, le germe de la 14 est morcelé en 4 à l’aide de la même instrumentation rotative suivant un tracé en croix. Cela permet d’optimiser l'économie en tissu osseux et de préserver la 54 sur arcade comme souhaité.

f: La cavité osseuse est soigneusement curetée afin de favoriser l'arrivée d'un caillot sanguin la comblant complètement.

$\mathrm{g}$ : Le lambeau est finalement suturé dans sa position initiale sans tension. Les conditions pour une bonne cicatrisation osseuse et une bonne cicatrisation muqueuse sont réunies. Un traitement antalgique et antiseptique est prescrit. Les suites opératoires ont été simples. Les autres germes seront extraits selon la même technique opératoire. 

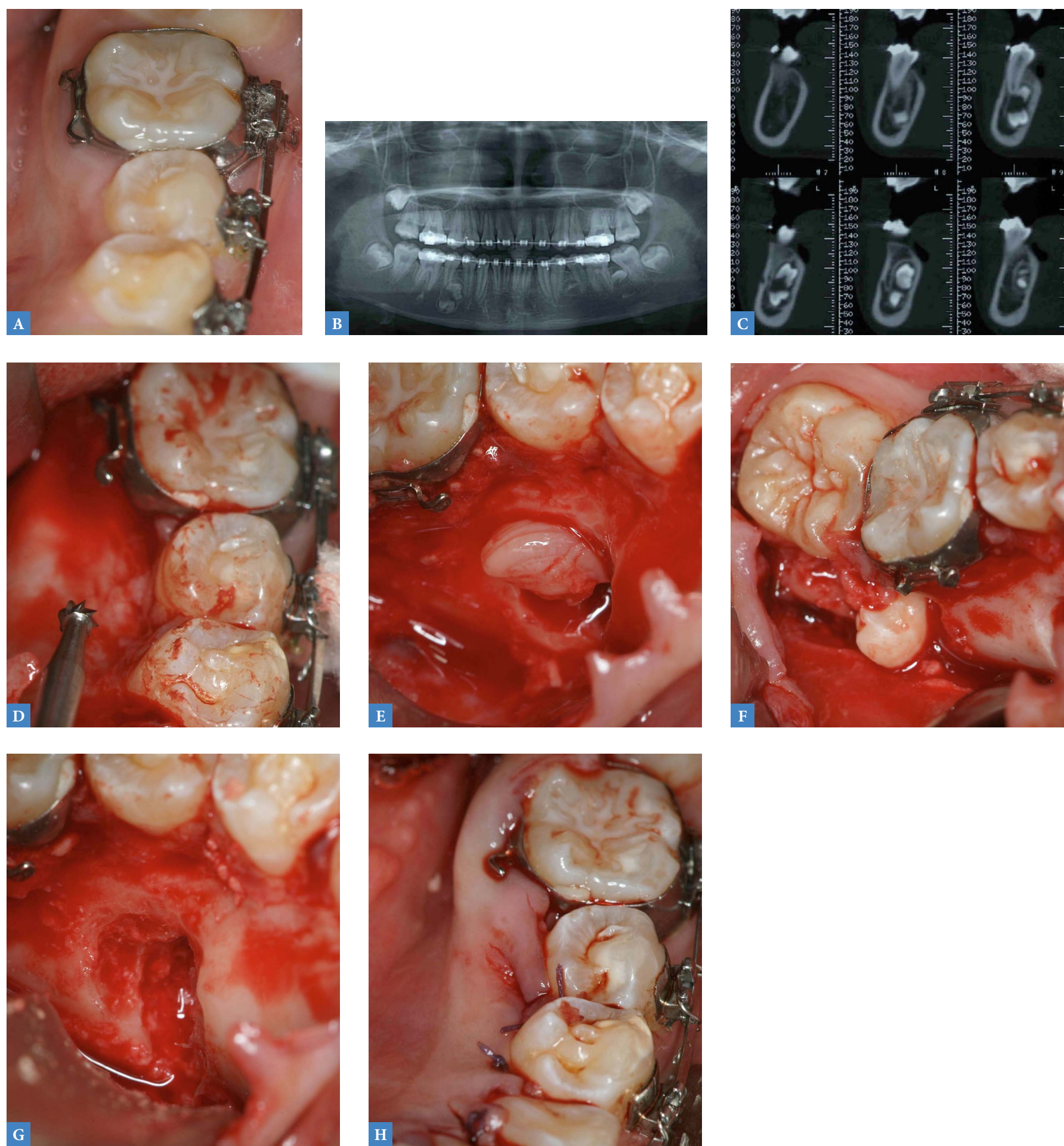

\section{$\triangle$ Figure 2}

a : Cette jeune patiente de 15 ans est adressée par son orthodontiste afin d'extraire tous les odontomes entravant le mouvement orthodontique. Leur découverte est récente car ils n'étaient pas visibles sur le cliché de début de traitement.

b : Le cliché panoramique objective la présence de plusieurs odontomes mandibulaires entre 44 et 45 , entre 34 et 35 et entre 36 et 37 . Il est décidé de commencer par le secteur 4 où siègent ceux qui semblent le plus délicat à retirer.

c: Un cone beam complémentaire permet de bien visualiser ces deux odontomes tête-bêche au niveau du foramen mentonnier bien discerné. Une voie d'abord vestibulaire est dès lors proscrite. De plus, ces odontomes sont collés contre la corticale linguale, l'ouverture buccale optimale et cette patiente semble coopérative. Une antibioprophylaxie est prescrite. L'intervention se déroulera sous anesthésie loco-régionale.

d : Une incision intrasulculaire linguale de grande étendue est pratiquée et un lambeau de pleine épaisseur récliné assez profondément sans désertion du mylohyoïdien. Une trépanation osseuse est pratiquée à la fraise boule acier montée sur pièce à main entre la 44 et la 45 .

e : L'odontome supérieur est rapidement visualisé puis extrait.

f : Puis, l'odontome inférieur est à son tout extirpé.

g: La cavité osseuse est curetée et l'arrivée du caillot sanguin soigneusement contrôlée.

$\mathbf{h}$ : Le lambeau lingual est ensuite suturé dans sa position initiale à l'aide de points interdentaires. Un traitement antalgique et antiseptique est prescrit. Les suites opératoires ont été simples. Les autres odontomes dans le secteur 3, beaucoup plus superficiels, ont été extraits par voie vestibulaire. 

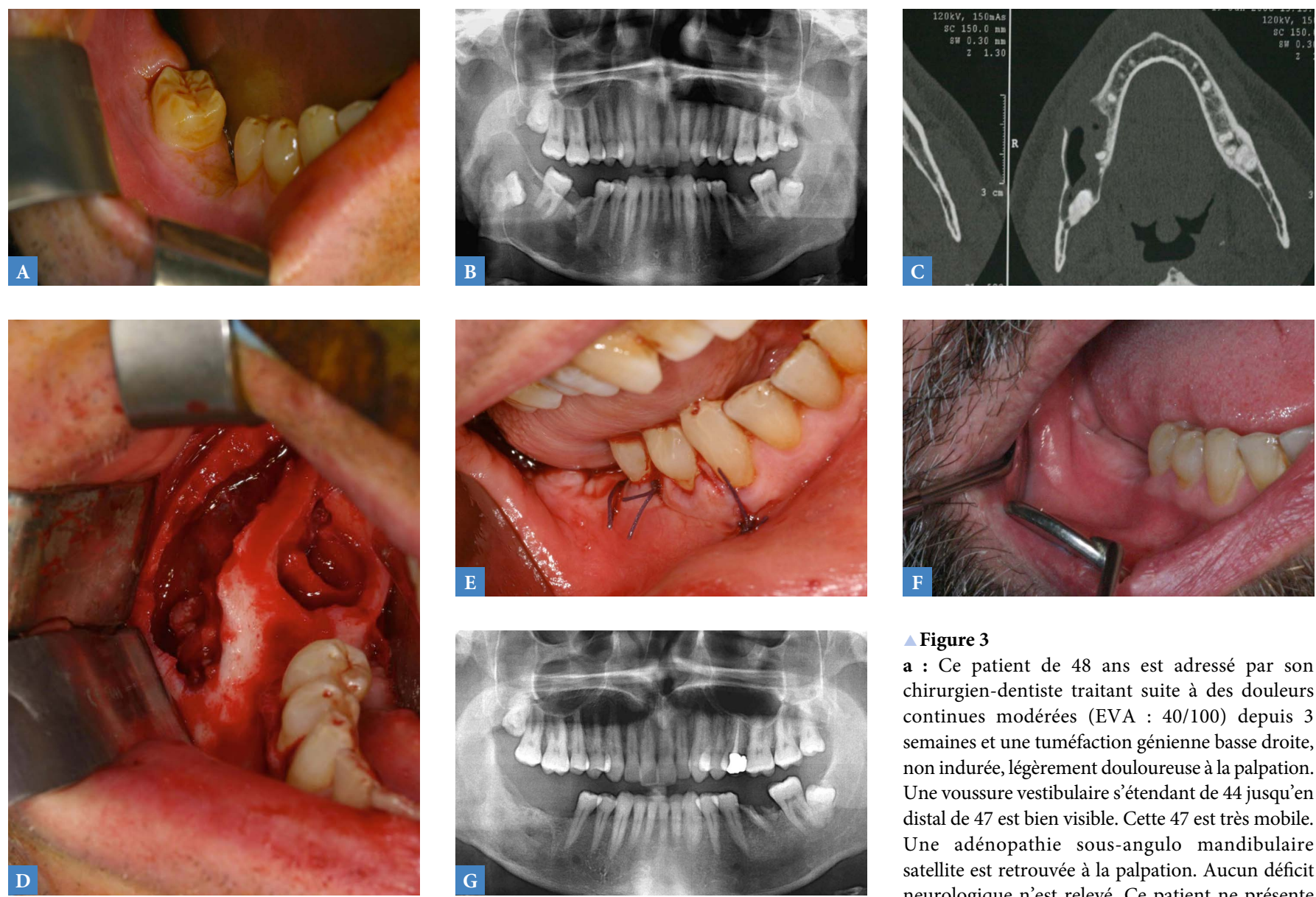

$\triangle$ Figure 3

a : Ce patient de 48 ans est adressé par son chirurgien-dentiste traitant suite à des douleurs continues modérées (EVA : 40/100) depuis 3 semaines et une tuméfaction génienne basse droite, non indurée, légèrement douloureuse à la palpation. Une voussure vestibulaire s'étendant de 44 jusqu'en distal de 47 est bien visible. Cette 47 est très mobile. Une adénopathie sous-angulo mandibulaire satellite est retrouvée à la palpation. Aucun déficit neurologique n'est relevé. Ce patient ne présente aucun antécédent médico-chirurgical notable.

b : Le cliché panoramique révèle une large image lacunaire mandibulaire postérieure s'étendant de la 44 jusqu'au milieu de la branche montante. La 48 est incluse dans cette image et des reliquats radiculaires de 46 sont bien visibles.

c: Une imagerie tomodensitométrique permet un bilan d'extension précis de cette image. On peut noter une rupture de la corticale vestibulaire. Le diagnostic de kyste dentigère est retenu en première intention bien qu'un kératokyste ou un améloblastome ne puissent être exclus d'emblée.

d : Une énucléation avec extraction de la 47, de la 48 et des reliquats radiculaires de 46 est réalisée sous anesthésie générale. Un large lambeau vestibulaire mucopériosté a permis un accès aisé à la lésion. Une antibiothérapie associée à une corticothérapie a été prescrite au préalable puis prolongée pendant une semaine (la corticothérapie a été stoppée après 5 jours). La pièce opératoire est envoyée à l'examen anatopathologique.

e : Le lambeau est repositionné et suturé dans sa situation initiale. Le patient est sorti le lendemain de l’intervention sans complication notable, en particulier neurologique. Une alimentation mixée est recommandée pendant 3 semaines de manière à prévenir tout risque de fracture.

f : Un mois après l'intervention, aucune suite opératoire n'est à déplorer, ni infectieuse, ni fracturaire, ni neurologqiue. La cicatrisation muqueuse est optimale. L'adénopathie sous anulo-mandibulaire satellite n'est plus perceptible. L'examen histologique a conclu à un kyste dentigère aux dépends de la 48 incluse. $\mathrm{g}$ : 9 mois après l'intervention, la ré-ossification de la lésion lacunaire est complète.
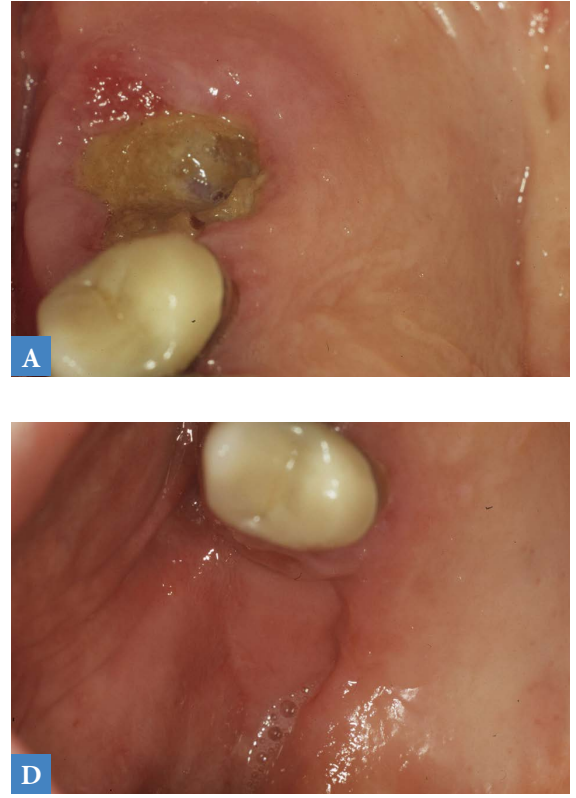
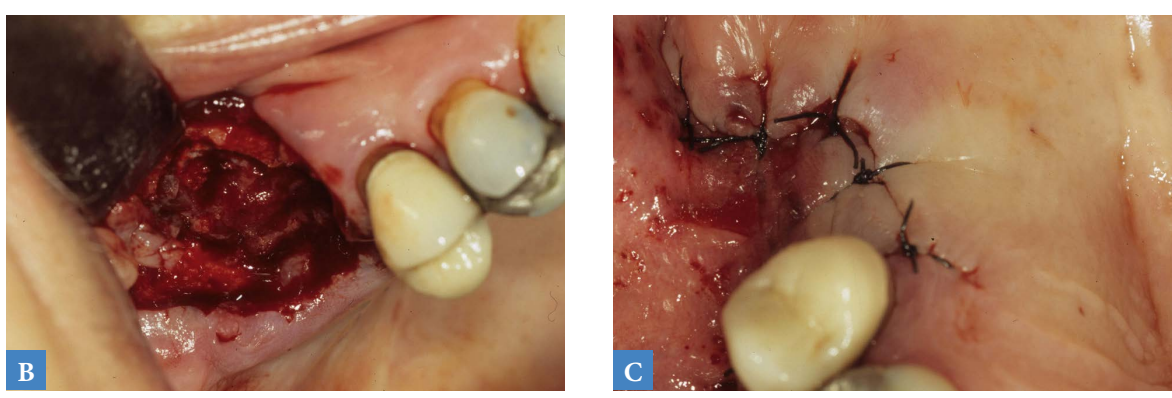

Figure 4 :

a : Cette patiente atteinte d'un myélome multiple traitée par bisphosphonates IV depuis deux présente une ostéonécrose des maxillaires révélée par l'extraction d'une première molaire maxillaire il y a 3 mois. Son praticien traitant l'a placée sous traitement antibiotique (amoxicilline + métronidazole) et l'a adressée pour une prise en charge hospitalière.

b : Une ostéoectomie (et non une séquestrectomie) est pratiquée sous anesthésie locale visant simplement à éliminer l'os nécrotique exposé en bouche et surinfecté.

c: Le lit osseux est ensuite recouvert d'une couche de colle biologique à base de fibrine puis le lambeau est suturé sans tension par dessus le site opératoire. Les suites opératoires sont simples. La patiente est toujours sous antibiotique. Un traitement antalgique et antiseptique est prescrit

d: La cicatrisation muqueuse acquise en première intention en 15 jours (l'antibiothérapie est alors stoppée) persiste un an plus tard. Ce cas illustre la pertinence d'une prise en charge chirurgicale dans ce type d'ostéonécrose. 

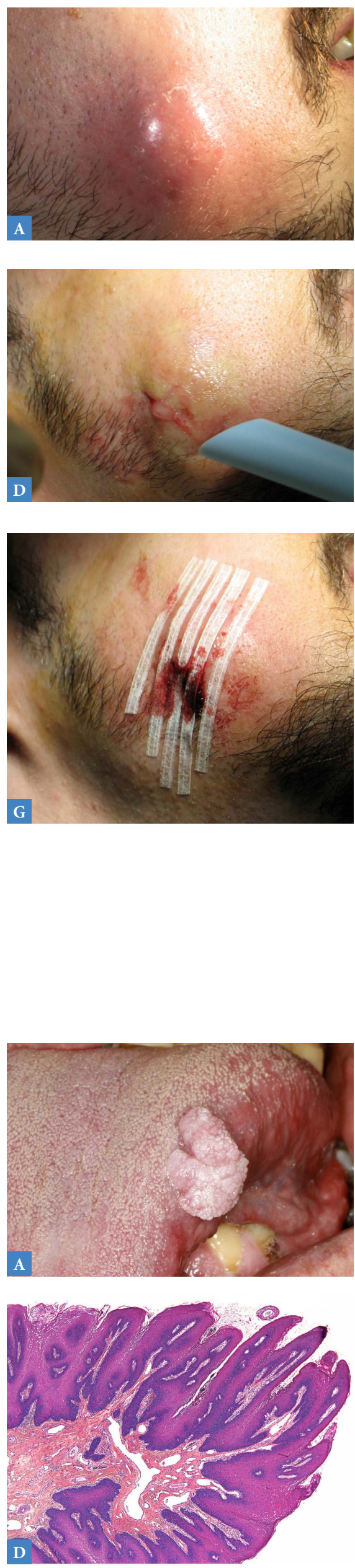
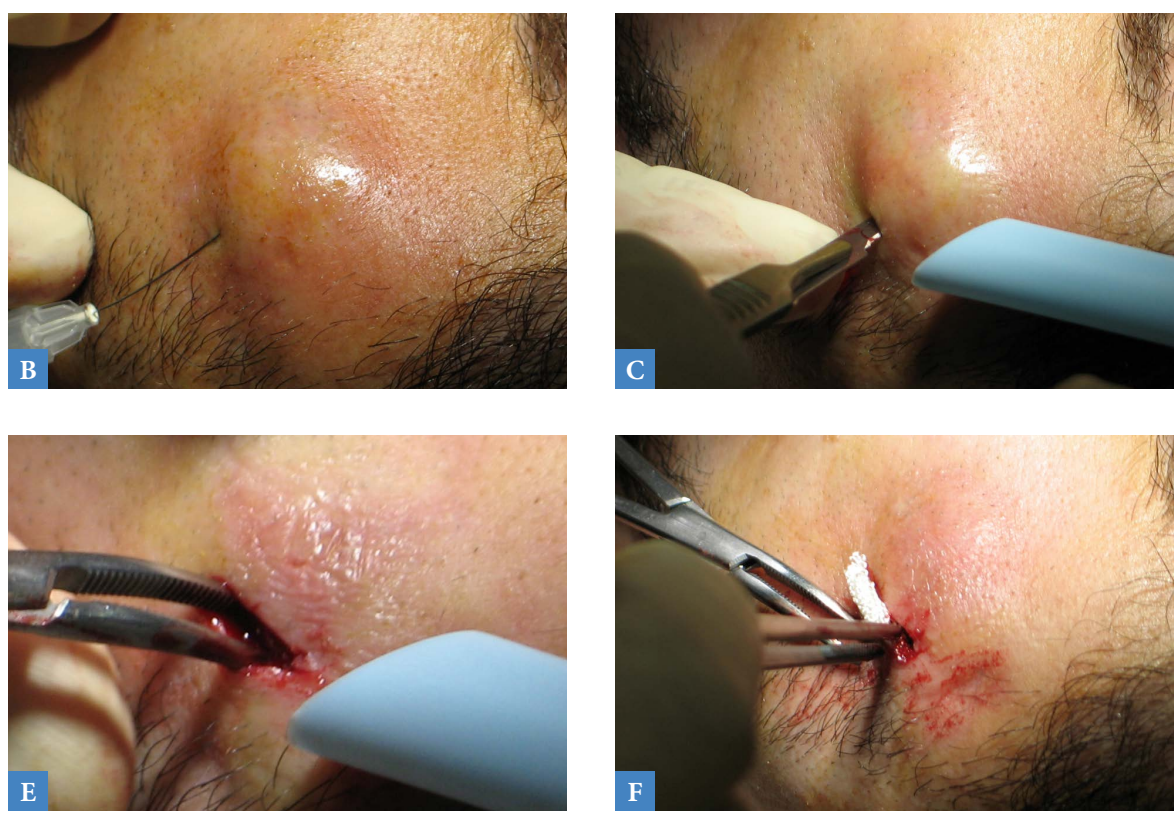

\section{$\triangle$ Figure 5}

a : Ce jeune patient s'est présenté aux urgences pour une cellulite génienne basse droite en rapport avec une 46 délabrée. Cette vue permet de nettement identifier une cellulite collectée. Ce patient ne présente aucun signe de gravité sur le plan général. Il s'est automédiqué depuis le matin avec de l'amoxicilline et du paracétamol pour des douleurs modérées (EVA : 40/100). Il est décidé de pratiquer un drainage par voie cutané.

b : Après une désinfection de surface, une anesthésie locale du revêtement cutané est pratiquée.

c: Puis une incision cutanée est réalisée à la partie la plus déclive de la collection .

d : permettant de drainer une partie de la collection.

e : L'ouverture est bien débridée de manière à favoriser l'évacuation d'une quantité maximale de pus et f : de manière à placer une compresse qui servira de drain.

g : Un pansement cutané clôture la prise en charge en urgence. La prescription d'amoxicilline et de paracétamol est prolongée. Trois jours plus tard, ce pansement sera déposé, le drain retiré et la 46 extraite. Tout rentre dans l'ordre en une semaine.
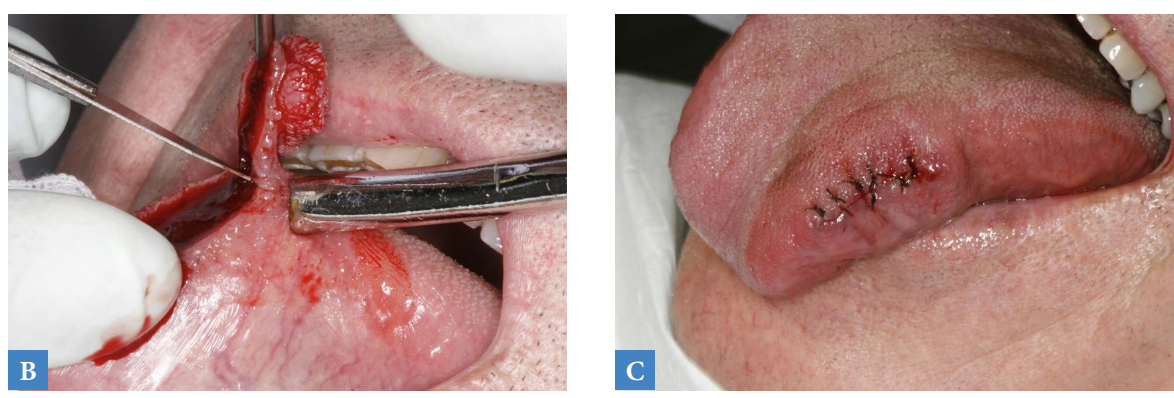

\section{$\triangle$ Figure 6}

a : Ce patient présente une lésion muqueuse hypertrophique sur le bord gauche de la langue mobile. Sa surface apparaît kératosique. Sa palpation est indolore et ne retrouve aucune induration sous-lésionnelle. Aucune adénopathie satellite n'est perçue. Cette lésion est totalement asymptomatique. Aucune étiologie traumatique ne peut être avancée. Un diagnostic de papillome ou de condylome est alors avancé.

b : Une exérèse de la lésion est pratiquée sous anesthésie locale. L'incision circulaire est réalisée au pied de la lésion, en zone muqueuse cliniquement saine. La pièce opératoire est envoyée à l'examen anatomopathologique.

c: Des points de suture séparés viennent fermer hermétiquement la zone opératoire. Une prescription antalgique et antiseptique est réalisée. Les suites opératoires ont été simples. Une semaine plus tard, ces points seront déposés, la cicatrisation muqueuse étant optimale.

d: L'examen histologique confirme le diagnostic de papillome. 


\section{LE DIPLÔME D'ÉTUDES SUPÉRIEURES DE CHIRURGIE ORALE (DESCO)}

Ce diplôme a vu sa naissance officielle au travers du décret 2011-22 du 5 janvier 2011 relatif à l'organisation du troisième cycle long des études en Odontologie et de l'arrêté du 31 mars suivant du ministère de l'enseignement supérieur et de la recherche fixant la liste des formations qualifiantes et la réglementation des diplômes spécialisés en Odontologie publiés au Journal Officiel de la République Française. Les premiers internes à s'être inscrits en DESCO ont commencé leur cursus le $1^{\text {er }}$ novembre de la même année. Comme il s'agit d'un parcours regroupant 8 semestres à temps plein, les premiers étudiants diplômés en chirurgie orale achèveront ce cursus le 31 octobre 2015. Le DESCO se substitue à l'ancien DES de stomatologie et au DESCB.

Ce diplôme est vraiment original puisqu'il possède une double entrée, médicale par l'intermédiaire de l'examen classant national et dentaire par celui de l'internat en Odontologie. Actuellement, 15 postes d'internes en chirurgie orale sont ouverts chaque année par chacune des filières soit au total 30 postes d'internes DESCO par an.

L'enseignement dispensé aux étudiants de DESCO garantit l'homogénéité de ce diplôme malgré différentes filières de provenance. Il comprend trois parties :

I Une formation théorique complémentaire en odontologie pour les internes provenant de la filière médecine et en médecine pour les internes provenant de la filière dentaire. Cette formation comporte 7 modules dispensés sous forme de séminaires au cours des 4 premiers semestres.

I Une formation théorique en chirurgie orale commune aux deux filières de 8 modules sous forme de séminaires.

I Une formation pratique de 8 semestres dont 3 semestres en chirurgie orale, 3 semestres en chirurgie maxillo-faciale et 2 semestres au choix dans des services hospitaliers agréés.

L'obtention du diplôme est soumise aux validations sous forme d'examens écrits et oraux de ces formations, aux validations des stages hospitaliers semestriels et à la soutenance d'un mémoire. Il est également prévu et recommandé que ces internes puissent exercer une activité de recherche durant ce cursus de manière à pouvoir constituer un vivier pour le recrutement des futurs enseignants hospitalo-universitaires et des futurs praticiens hospitaliers de cette discipline.

\section{LES COMPÉTENCES À ACQUÉRIR}

La chirurgie orale est une discipline médico-chirurgicale dont le champ de compétences est parfaitement défini dans le référentiel activités-compétences-formation relatif à l'internat qualifiant en chirurgie orale. Ce champ s'articule autour de deux grands thèmes, les compétences médicales et les compétences chirurgicales.

\section{Compétences chirurgicales}

\ous types d'avulsions dentaires,

Les transplantations dentaires autogènes,

$\checkmark$ La chirurgie du péri-apex dentaire,

I Le traitement chirurgical des kystes odontongènes et non odontogènes des maxillaires,

I La chirurgie alvéolaire à visée orthodontique (à l'exclusion donc de la chirurgie des bases osseuses), I Les traumatismes alvéolo-dentaires,

$\checkmark$ Le traitement chirurgical des fractures des bases maxillaire et mandibulaire accessibles par voie endo-buccale seule,

\Les urgences chirurgicales en particulier les urgences infectieuses et les urgences hémorragiques,

I Le traitement chirurgical des communications bucco-sinusiennes et bucco-nasales,

La chirurgie implantaire, pré-implantaire (en particulier les prélèvements osseux intra-buccaux) et péri-implantaire,

I La chirurgie pré-prothétique intéressant les tissus minéralisés comme les tissus muqueux, avec recours à tous types de biomatériaux autorisés, prélèvements extra-buccaux exclus,

I La chirurgie muqueuse endo-buccale des lésions et/ ou tumeurs bénignes,

La chirurgie salivaire des lithiases et/ou des tumeurs bénignes par voie endo-buccale.

\section{Compétences médicales}

I La prise en charge des patients ayant ou devant bénéficier d'une radiothérapie cervico-faciale,

$\checkmark$ La prise en charge des patients ayant une pathologie et/ou une thérapeutique intercurrente,

I La pathologie de la muqueuse buccale des lésions et/ ou tumeurs bénignes,

ILe dépistage des lésions et des tumeurs malignes de la cavité buccale, des maxillaires et de la mandibule, coordonner le suivi thérapeutique,

I L'évaluation et traitement des douleurs oro-faciales et les pathologies de l'appareil manducateur (hors chirurgie temporo-mandibulaire),

$\checkmark$ Les urgences médicales en particulier la prise en charge des différents types de malaise,

I La pathologie salivaire bénigne par voie endobuccale seule. 
Les internes DESCO acquièrent ces compétences médico-chirurgicales dans des structures agréées, principalement les services ou unités fonctionnelles de chirurgie orale des services hospitaliers d'Odontologie et les services hospitaliers de Chirurgie maxillo-Faciale des différents $\mathrm{CHU}$ français ou établissements publics hospitaliers. L'agrément est délivré par les Agences Régionales de Santé.

\section{L'EXERCICE DE LA CHIRURGIE ORALE}

Pour les chirurgiens dentistes, la qualification en chirurgie orale est l'apanage d'une commission nationale ad hoc sous l'égide du conseil national de l'ordre des chirurgiens dentistes. Cette commission comprend actuellement :

I 3 Professeurs des Universités-Praticiens Hospitaliers assurant l'enseignement de la spécialité

11 représentant du Syndicat National des Chirurgiens Oraux

11 représentant du Syndicat national des Odontologistes des Hôpitaux Publics

I 1 représentant du Conseil National de l'Ordre des Chirurgiens Dentistes

Il existe une procédure de recours en cas d'avis négatif. Une fois la qualification prononcée, l'inscription sur la liste des praticiens qualifiés en chirurgie orale est du ressort du praticien lui même. A partir du moment où le praticien qualifié est inscrit sur cette liste, il s'engage à exercer exclusivement la chirurgie orale selon le champ de compétence figurant dans le référentiel relatif à l'internat qualifiant en chirurgie orale.

La qualification en Chirurgie Orale est accordée aux titulaires du DESCO. Cette commission examine régulièrement les demandes de qualification émanant de praticiens non titulaires du DESCO mais pouvant faire état de compétences recouvrant celles du référentiel du DESCO, en particulier pour les titulaires du DESCB. Pour les médecins, une telle commission de qualification n'est pas encore constituée.

\section{CONCLUSION}

De fait, ces nouveaux praticiens qualifiés en chirurgie orale vont d'une part remplacer les médecins stomatologistes du fait de la fermeture du DES de Stomatologie et d'autre part succéder aux chirurgiens dentistes titulaires du DESCB. Ce nouveau diplôme va permettre après une formation croisée des médecins vers l'odontologie et des chirurgiens dentistes vers la médecine, de former des praticiens ayant un champ d'activité élargi de la médecine orale et de la chirurgie orale. Une nouvelle ère s'ouvre donc pour cette spécialité qui va se nourrir de ses deux filières d'origine, qui va murir et qui va proposer une offre de soins de haute qualité (comme l'illustrent les cas cliniques de cet article, tous réalisés par des étudiants de spécialité) dans un secteur très déficitaire.

\section{DOCUMENTS ET SITES UTILES}

Arrêté du 31 mars 2011 :

http://www.legifrance.gouv.fr/affichTexte.do,cidTetxte+JORFTEXT000023877522\&dateTexte=\&categorielLien=id

Association Nationale des Internes en chirurgie Orale :

www.anico.fr

Décret 2011-2 du 5 janvier 2011 :

http://www.legifrance.gouv.fr/affichTexte.do,cidTetxte+JORFTEXT000023375670\&dateTexte=\&categorielLien=id

\section{Diplôme d'Études Supérieures de Chirurgie Orale :}

www.desco.fr

Syndicat national des chirurgiens oraux :

www.snco.fr 